\title{
Saving Our Healthcare Workers Again During the COVID-19 Pandemic in the Philippines
}

\author{
Youngrong Lee', Sun Jae Jung ${ }^{1,2}$ \\ ${ }^{1}$ Department of Preventive Medicine, Yonsei University College of Medicine, Seoul, Korea; ${ }^{2}$ Department of Public Health, Yonsei University Graduate \\ School, Seoul, Korea
}

\section{Dear Editor,}

This letter brought up the issue of improving social awareness of healthcare workers (HCWs) who are suffering from an increased workload in the ongoing coronavirus disease 2019 (COVID-19) pandemic. The author explained the duties performed by HCWs in the Philippines during COVID-19 and the maltreatment they have suffered outside of the workplace. Even taking into account the different situations of HCWs in each country, we are concerned by the current adverse experiences of medical personnel and the stigma placed on them in the Philippines. Medical professionals in many countries endure work-related difficulties caused by the pandemic, such as shortages of protective gear [1], and they also undergo stress caused by their increased workload and worries about infection, which can lead to an increased prevalence of posttraumatic stress disorder (PTSD), anxiety, and depression [2]. Nevertheless, in this urgent situation, medical professionals perceive themselves as not receiving adequate compensation or appropriate attention from their own government [3]. The author argued that a national and social project should be implemented for medical professionals that would include guaranteed salaries, the provision of protective equipment in a timely

\section{Corresponding author: Sun Jae Jung}

Department of Preventive Medicine, Yonsei University College of

Medicine, 50-1 Yonsei-ro, Seodaemun-gu, Seoul 03722, Korea

E-mail: sunjaejung@yuhs.ac

This is an Open Access article distributed under the terms of the Creative Commons Attribution Non-Commercial License (https://creativecommons.org/licenses/bync/4.0// which permits unrestricted non-commercial use, distribution, and reproduction in any medium, provided the original work is properly cited. manner, and campaigns or public advertisements exalting HCWs working at the forefront of quarantine to prevent the healthcare system from collapsing due to burnout and withdrawal of HCWs.

We fully agree with the author's argument that appropriate compensation and awareness of HCWs' work should be implemented. Taking such steps will stabilize the medical system in this global crisis of the COVID-19 pandemic and will lead to a more sustainable and efficient response. As the author points out, compensation due to the increased working hours and exhausting work environment should be provided in a timely manner.

However, we consider that the definition of "unsung heroes" who respond to this crisis does not only apply to medical personnel, but should be expanded to include the public service and private sectors in their entirety. Our previous research pointed out that non-medical personnel in the hospital workplace setting were more likely to have PTSD symptoms than medical personnel [4]. The current quarantine does not only depend on the work of medical personnel in the strict sense of the term, but also relies on the dedication of workers in other fields in society as a whole. Our study implies the need for a support system covering the entire society that goes beyond protective measures for the well-being of medical personnel. We would like to appreciate all these workers and consider hardships beyond a single specific sector to create a sustainable medical system.

In order to prevent burnout of HCWs and create a satisfactory workplace environment, we would like to recommend developing an evidence-based decision-making environment in 
workplaces, appropriately distributing the workload, providing reasonable monetary compensation and guaranteed days off, and utilizing the idle healthcare workforce who are not working on active duty. Monitoring mental health along with physical health and implementing lifestyle changes in these focused areas would also be warranted [4]. It is believed that such a proposal should be implemented not only for medical personnel, but also for all workers in all public response systems responding to the pandemic.

According to the results of our study, previous Middle East respiratory syndrome experience was associated with an increase in PTSD symptoms among hospital workers who were currently experiencing the COVID-19 pandemic [2]. In the future, when the current pandemic will come to an end, it is possible that the outbreak of a new pandemic may provoke an aggravating impact on the mental health of HCWs who have experienced the adversity of COVID-19 (possibly including non-medical personnel). A new standard in preparation for the resurgence of infectious diseases will be necessary for our society, and it is necessary to develop mental health protection services including regular population monitoring and psychological interventions for medical personnel, as well as to introduce public health administrative governance structures to facilitate a flexible response to sudden changes in the workload and workplace environment of HCWs [5].

\section{CONFLICT OF INTEREST}

The authors have no conflicts of interest associated with the material presented in this paper.

\section{ORCID}

Youngrong Lee https://orcid.org/0000-0003-1367-4381

Sun Jae Jung https://orcid.org/0000-0002-5194-7339

\section{REFERENCES}

1. Minder R, Peltier E. Virus knocks thousands of health workers out of action in Europe. New York Times; 2020 Mar 24 [cited 2020 Apr 27]. Available from: https://www.nytimes.com/2020/ 03/24/world/europe/coronavirus-europe-covid-19.html.

2. Lee Y, Kim K, Park S, Jung SJ. Associations between general perceptions of COVID-19 and posttraumatic stress disorder in Korean hospital workers: effect modification by previous Middle East respiratory syndrome coronavirus experience and occupational type. J Prev Med Public Health 2021;54(2):86-95.

3. Chen R, Sun C, Chen JJ, Jen HJ, Kang XL, Kao CC, et al. A largescale survey on trauma, burnout, and posttraumatic growth among nurses during the COVID-19 pandemic. Int J Ment Health Nurs 2021;30(1):102-116.

4. Jung SJ, Jun JY. Mental health and psychological intervention amid COVID-19 outbreak: perspectives from South Korea. Yonsei Med J 2020;61(4):271-272.

5. Li Z, Ge J, Yang M, Feng J, Qiao M, Jiang R, et al. Vicarious traumatization in the general public, members, and non-members of medical teams aiding in COVID-19 control. Brain Behav Immun 2020;88:916-919. 